\title{
Gas-Phase Studies of Valinomycin-Alkali Metal Cation Complexes: Attachment Rates and Cation Affinities
}

\author{
Philip S. H. Wong, Barbara J. Antonio, and David V. Dearden \\ Department of Chemistry, The University of Texas at Arlington, Arlington, Texas, USA
}

\begin{abstract}
Reactions of laser-desorbed $\mathrm{Na}^{+}, \mathrm{K}^{+}, \mathrm{Rb}^{+}$, and $\mathrm{Cs}^{+}$with thermally vaporized valinomycin generate metal-ligand complexes in a Fourier transform ion cyclotron resonance trapping cell, proving that complexes can form via gas-phase ion-molecule reactions. Although desorption of intact pre-formed complexes cannot be ruled out, this route appears minor. Relative rate constants for the complexation reactions show strong dependence on the charge densities of the cations. Competition experiments between valinomycin and the synthetic ionophores 18-crown-6 (18C6) and [2.2.2]-cryptand ([2,2,2]) show that valinomycin has a higher intrinsic alkali metal cation affinity than either $18 \mathrm{C} 6$ or [2.2.2], in contrast to the complex formation constants observed in methanol, where $\mathrm{K}^{+}$affinities are in the order [2.2.2] > 18C6 > valinomycin. (f Am Soc Mass Spectrom 1994, 5, 632-637)
\end{abstract}

W ith the development of new ionization techniques such as matrix-assisted laser desorption [1] and electrospray [2], the role of mass spectrometry in biological research has profoundly expanded. Because both methods often produce alkali metal-attached molecular ions, the process of alkali cation attachment has also taken on new importance. Additionally, alkali cations such as $\mathrm{Li}^{+}, \mathrm{Na}^{+}$, and $\mathrm{K}^{+}$ have been shown to be very useful chemical ionization reagents [3-5]. A clear understanding of what factors govern cation binding strengths and binding site preferences is crucial for obtaining structural information from the mass spectra of large cationized molecules.

Until recently, most studies of alkali cation attachment have focused on binding to bases with only one donor site. However, for large molecules such as peptides, it is likely that multidentate attachment, through several interacting donor groups, is more important $[4,5]$. In ligands capable of multidentate binding, binding strengths can be very different from those expected based on comparison with unidentate ligands $[3,6]$. Hence, it is important to understand multidentate ligation in the gas phase.

We [7-10] and others [11-15] have used crown ethers as model compounds for the investigation of multidentate alkali cation binding in the gas phase. These ionophoric ligands, which show remarkable cation binding selectivity in solution, exhibit equally interesting gas-phase chemistry. For example, size rela-

Address reprint requests to Dr. David V. Dearden, Brigham Young University, Department of Chemistry, Box 24672, 226 Fyring Science Center, Provo, UT 84602-4672. tionships between the cations and the cavities of the ligands can strongly influence both the kinetics and thermodynamics of the binding interactions $[7-9,14]$. In this paper we report some initial attempts to extend our studies to include larger naturally occurring ionophores, such as valinumycin, which are of greater analytical and biological interest than the simple synthetic ligands we have examined heretofore. Valinomycin is a good choice for this extension because it is similar to the crown ethers in cation binding function and because it is well studied in solution, offering the possibility of gas-phase comparison studies for understanding solvation effects.

Valinomycin is a cyclic depsipeptide, built from a threefold repetition of the alternating amino acid and hydroxy acid residues L-valine, $D$ - $\alpha$-hydroxyvaleric acid, D-valine, and L-lactic acid. It functions as an antibiotic by influencing cation transport across cell membranes. Binding constants measured in methanol indicate valinomycin selectively binds $\mathrm{Rb}^{+}$in preference to the other alkali metal ions $[16,17]$. Its selectivity for $\mathrm{K}^{+}$over $\mathrm{Na}^{+}$is especially well established [18]. It has been proposed that the selectivity of valinomycin for $\mathrm{K}^{+}$over $\mathrm{Na}^{+}$correlates with the size of the binding cavity formed by the cyclic ionophore [19]. Theoretical studies have shown that the selectivity displayed by valinomycin with respect to the binding of alkali metal cations is a result of a trade-off between the interaction energies of this macrocyclic carrier with the alkali metal cations and the corresponding hydration energies of the cations and the ligand $[20,21]$. Gas-phase experiments are important because they directly probe the intrinsic selectivity of the ligand, in the absence of interfering solvation effects. 
A number of gas-phase studies involving valinomycin have appeared. A cross section for proton transfer from protonated valinomycin to ammonia of $2.4 \pm$ $1.2 \times 10^{-17} \mathrm{~cm}^{2}$ molecule $^{-1}$, at a collision energy of $26.5 \mathrm{eV}$ in the center-of-mass frame, has been measured, along with an estimated cross section for collision-induced dissociation $\left(\mathrm{CID}\right.$; with $\mathrm{ND}_{3}$ as the neutral target), of $2.0 \times 10^{-14} \mathrm{~cm}^{2}$ molecule ${ }^{-1}$ [22]. The very small cross section for proton transfer probably reflects steric or orientational requirements for the reaction.

Thermally vaporized valinomycin has been examined by using methane chemical ionization (CI) in a Fourier transform ion cyclotron resonance (ICR) instrument [23]. The $\left[\mathrm{M}+\mathrm{H}^{+}\right.$peak at $m / z 1111.6$ dominates the mass spectrum under low-pressure $\mathrm{CI}$ conditions. I nw energy CID in these experiments yielded a number of structurally significant product ions that give sequence information. Tandem mass spectrometry of fast-atom bombardment (FAB)-generated $[\mathrm{M}+\mathrm{K}]^{+}$ ions yields $\mathrm{K}^{+}$ions as the base peak in the spectrum, which suggests that loss of $\mathrm{K}^{+}$is energetically preferred over fragmentation of the ligand [24].

In this article we report relative rates for the attachment of alkali cations to valinomycin, which illustrate the importance of cation charge density in the complexation process. In addition, we compare the gasphase alkali cation affinities of valinomycin and comparable synthetic ionophores, 18-crown-6 and [2.2.2]cryptand, and contrast the results with data obtained in solution.

\section{Experimental}

Valinomycin-alkali metal cation complexes were readily generated and trapped in the gas phase by using laser desorption Fourier transform ion cyclotron resonance mass spectrometry. The solvent used for sample preparation in all experiments was absolute methanol. Valinomycin was dissolved in methanol, doped with an excess of the metal nitrate of interest, and deposited onto the tip of a stainless steel sample probe, where the solvent was evaporated. The loaded probe was inserted through a vacuum lock such that the tip was about $10 \mathrm{~cm}$ from the end of a capacitively coupled open ICR cell $[25,26]$. Samples were desorbed into the gas phase when the probe tip was irradiated with the focused output (1 to $5 \mathrm{~mJ}$ pulse ${ }^{-1}$ ) from a Lambda Physik. (Acton, MA) LPX 205i/FL 3002 excimerpumped dye laser $(\lambda=360 \mathrm{~nm})$. Experiments were conducted with a modified Millipore/Extrel (Madison, WI) FTMS-1000 instrument, equipped with a 3-T superconducting solenoid magnet, which has been described previously $[9,10]$. Cell pressures, measured by using a Bayard-Alpert ionization gauge mounted external to the region of strong magnetic field, were typically 2 to $8 \times 10^{-8}$ torr. The mass scale of the instrument was calibrated using electron impact (70-
$\mathrm{eV}$ ) ionization of tris-(perfluorononyl)-s-triazine, which yields several suitable calibration peaks in the 970 to 1485 mass-to-charge ratio range. Medium resolution spectra $(\mathrm{m} / \Delta \mathrm{m}$ of 3000 to 4000$)$ were typically obtained with $64 \mathrm{~K}$ data points and a bandwidth of 127 $\mathrm{kHz}$ in heterodyne mode.

Spectra were obtained for complexes with $\mathrm{Na}^{+}, \mathrm{K}^{+}$, $\mathrm{Rb}^{+}$, and $\mathrm{Cs}^{+}$with sufficient resolution to resolve the ${ }^{13} \mathrm{C}$ isotope peaks. Both resolution and signal-to-noise ratio were improved by operating at low trapping voltages $(0.02$ to $0.2 \mathrm{~V})$. This is probably due to reduced space charge effects at low trapping potential. Laser desorption produces ions with a wide range of kinetic energies [27]. Low trapping potentials allow ions with large energies in the $z$-direction (along the magnetic field lines) to escape, retaining only ions with small kinetic energy in the $z$-direction. The low trapping potential therefore lowers the ion density in the trap and selects a more uniform distribution of kinetic energies. Both factors probably assist in ion detection [28]. Operation at low trapping voltage is probably more difficult in a closed cell: axial ejection of ions during excitation [29-31] in a conventional cubic cell can be quite severe at low trapping potential.

The relative intensities of the peaks matched well with the expected isotope distributions of the valinomycin-metal complexes. The observation of peaks at the expected mass-to-charge ratio values, along with the expected isotopic patterns, confirms the mass spectroscopic assignments. The error in mass-to-charge ratio is about $+0.05 \%$, which is rather large for a Fourier transform mass spectrometry mass determination. Mass scale calibration was done at a trapping potential of $1.0 \mathrm{~V}$, whereas the spectra were acquired at much lower trapping vollages $(0.02$ to $0.2 \mathrm{~V})$. The positive shift in mass-to-charge ratio with decreased trapping potential is contrary to expectations from theory [32]. Possibly the deviation is due to magnetic capacitors that were attached to the cell during the experiment. It is also possible that at very low trapping potentials the local electrostatic field in the vicinity of the ions, which leads to shifts in effective cyclotron frequency, is dominated by space charge rather than by the applied trapping field. If so, quantitative predictions about shifts in effective cyclotron frequency could not be made without detailed knowledge of the space charge effects.

In experiments designed to determine whether complexes were desorbed intact or formed in gas-phase ion-molecule reactions, the alkali metal nitrate was laser desorbed from the probe surface and valinomycin was introduced separately by thermal vaporization of a sample placed in a small capillary tube mounted in the center of the temperature-controlled probe. Temperatures of about $300{ }^{\circ} \mathrm{C}$ (measured with a thermocouple in the probe tip) were required. By using the same technique, the relative rates of metal complexation were investigated. In these experiments, two or more alkali metal nitrates were co-deposited on the 
probe and desorbed together, ensuring that each cation is exposed to the same pressure conditions in the cell. The intensities of the metal ion peaks, as well as those of the complex peaks, were followed as a function of time and analyzed assuming pseudo-first-order kinetic conditions. Corrections were made for metal ion losses arising from attachment to neutral species other than valinomycin, which were also present in the chamber. Because we were unable to measure absolute valinomycin partial pressures, only relative rate constants were obtained.

Relative complex stabilities were examined in competition experiments between valinomycin $(\mathrm{Vm})$ and the synthetic ionophores 18-crown-6 (18C6; Sigma Chemical Co., St. Louis, MO) and [2.2.2]-cryptand ([2.2.2]; Aldrich Chemical Co., Milwaukee, WI). Reactions (1) and (2) were each examined in both directions and ionic reactants were isolated by using standard Fourier transform ICR swept-rf methods [32], and products were monitored as a function of time:

$$
\begin{aligned}
V m+[2.2 .2] M^{+} & \rightleftharpoons V m M^{+}+[2.2 .2] \\
V m+18 C 6 M^{+} & \rightleftharpoons V m M^{+}+18 C 6
\end{aligned}
$$

\section{Discussion}

The structures of valinomycin, 18-crown-6, and [2.2.2]-cryptand are shown in Figure 1. Although we cannot rule out desorption of intact pre-formed metal complexes from mixtures of the ligand with metal salts, it is clear that the ommplexation can ocrur as a purely gas-phase reaction. Thermal evaporation of valinomycin, accompanied by laser desorption of alkali metal salts, resulted in the formation of metal complexes in the gas phase via ion-molecule reactions. Whereas the two reactants in this experiment cannot come in contact except in the gas phase, this clearly demonstrates that gas-phase complexation is possible. The mass spectra include some peaks assigned to valinomycin fragments. Fragments might arise from partial thermal decomposition of the ligand at the temper-
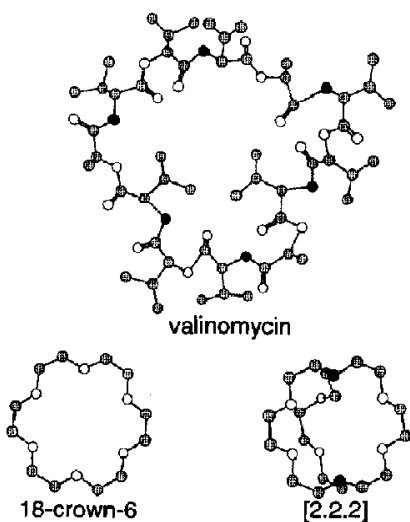

Figure 1. Structures of the ligands used in this study. ature required for evaporation. In addition, it may be that the energy released by the complexation reaction is sufficient to result in fragmentation.

The relative complexation rates with respect to potassium-complex formation are shown in Table 1. The relative rate constants decrease as the mass of the cation increases, and seem to correlate with collision rates calculated by using ion-induced dipole collision theory [33], which predicts an inverse dependence on the square root of the reduced mass of the collision pair. A close look at the data suggests, however, that the reactions are not collision controlled and that details of the metal-ligand interaction are probably important in determining relative rates.

The dipole moment of valinomycin in solution has been calculated and ranges up to $6.3 \mathrm{D}$ depending on the nature of the solvent system [34]. In a hydrocarbon solvent (dioxane-octane), valinomycin has a dipole moment of $3.9 \mathrm{D}$ [34]. We expect that valinomycin in the gas phase has a dipole moment similar to that in a nonpolar solvent. If we assume the complexation reaction is collision limited and use this dipole moment, average dipole orientation (ADO) theory [33] can be used to estimate absolute reaction rates and the valinomycin vapor pressure. Table 1 shows collision rates (both absolute and relative) estimated from theory. The estimated absolute rate constants are on the order of $10^{-9} \mathrm{~cm}^{3}$ molecule $\mathrm{s}^{-1}$. From these data the valinomycin vapor pressure is estimated to be approximately $10^{-9}$ torr, on the same order as the baseline pressure of our instrument.

It is very interesting to note, from the last column of Table 1, that the reaction efficiencies decrease as the size of the metal cation increases (i.e., the reaction rates vary by more than the amount of variation predicted for the collision rates). If all the reactions really are collision controlled, the data could be explained if approaches such as ADO theory are not adequate for describing large-molecule systems such as this one. For large molecules like valinomycin, the assumption that the colliding ion and molecule are point particles (implicit in polarization theory) may break down. However, the collision cross section estimated by using Langévin theory [33] is about 920 to $960 \AA^{2}$, whereas the physical cross section of valinomycin estimated from $x$-ray data [35] is about $77 \AA^{2}$. Even for a molecule as large as valinomycin, the Langévin cross section is much larger than the physical extent of the molecule. It is unlikely that the size of valinomycin accounts for large failures in collision theory.

A much more likely explanation is that the complexation reactions are not collision controlled and that the decrease in reaction efficiency with increasing cation size is real. It seems plausible that the higher charge density for the smaller cations may lead to greater ion-dipole interactions with the ligand, inducing more rapid rearrangement of the ligand into a conformation favorable for binding. These kinds of effects have been postulated to explain similar trends in the efficiencies of alkali cation complexation by 
Table 1. Relative experimental rate constants (normalized to values for $\mathrm{K}^{+}$) and calculated collision rate constants for the formation of valinomycin-alkali metal ion complexes

\begin{tabular}{|c|c|c|c|c|}
\hline \multirow[b]{2}{*}{ Cation } & \multirow{2}{*}{$\begin{array}{l}\text { Relative } \\
\text { rate } \\
\text { constant }\end{array}$} & \multicolumn{2}{|c|}{ Estimated Collision Rate Constants } & \multirow{2}{*}{$\begin{array}{l}\text { Relative } \\
\text { reaction } \\
\text { efficiency }\end{array}$} \\
\hline & & $\begin{array}{c}\text { Absolute } \\
\left(\times 10^{9} \mathrm{~cm}^{3} \text { molecule }{ }^{-1} \mathrm{~s}^{-1}\right)\end{array}$ & Relative & \\
\hline$\overline{\mathrm{Na}^{+}}$ & $1.9 \pm 0.4$ & 6.5 & 1.3 & $1.5+0.3$ \\
\hline $\mathrm{K}^{+}$ & 1 & 5.0 & 1 & 1 \\
\hline $\mathbf{R} \mathbf{b}^{+}$ & $0.31 \perp 0.03$ & 3.5 & 0.69 & $0.45 \pm 0.04$ \\
\hline $\mathrm{Cs}^{+}$ & $0.15 \pm 0.04$ & 2.8 & 0.59 & $0.25 \pm 0.07$ \\
\hline
\end{tabular}

crown ethers [9]. In fact, we have observed similar effects with every ligand we have investigated.

The transfer of alkali metal cations $\left(\mathrm{Na}^{+}, \mathrm{K}^{+}, \mathrm{Rb}^{+}\right.$, and $\mathrm{Cs}^{+}$) from two synthetic ionophores ([2.2.2] and 18C6) to valinomycin was observed. Figure 2 shows the transfer of $\mathrm{K}^{+}$from the [2.2.2]-cryptate complex to valinomycin as a function of reaction time. Both the decay of the reactant signal and growth of the product signal can be fitted with single exponential functions with the same rate constant, consistent with a pseudufirst-order reaction. The reverse reactions were not seen, even though the neutral vapor pressures of the synthetic ionophores were certainly much greater than that of valinomycin. The formation of heterogeneous 2:1 ligand:metal complexes involving valinomycin also was not observed, even at reaction times of several minutes. For simple crown ether ligands, this failure to observe 2:1 ligand:metal species was interpreted to indicate that the metal is effectively encapsulated in the $1: 1$ complexes, making it unavailable as a site to attach a second ligand $[7,9]$. For a large ligand such as valinomycin, it is not surprising that the cation might be enveloped by the neutral host molecule.

In other cation-ionophore systems where both the forward and reverse reactions were observed, equilibrium constants were determined [9]. The variation of equilibrium constant with cation size exhibited patterns that could be rationalized in terms of the selectiv-

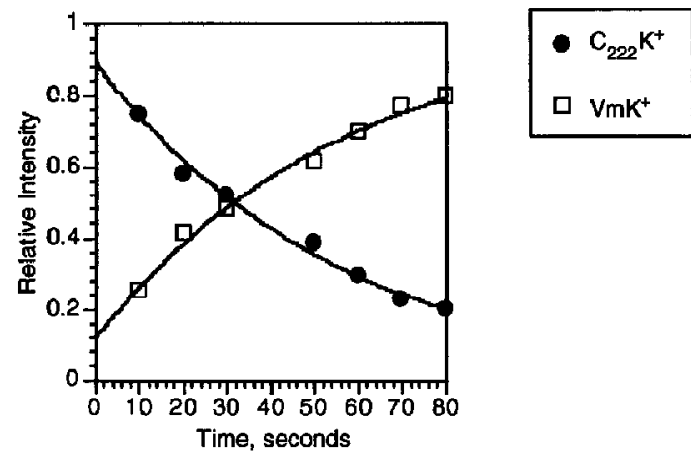

Figure 2. Relative intensities of valinomycin $-\mathrm{K}^{+}$and [2.2.2]- $\mathrm{K}^{+}$ complexes as a function of time, showing transfer of $\mathrm{K}^{+}$from [2.2.2]-cryptate complex to valinomycin. ity of the ligands for cations of a particular size. Unfortunately, failure to observe equilibrium in the valinomycin system precludes a detailed analysis of selective cation binding in this system, although on the basis of comparison to the functionally similar crown ethers we believe it likely that such selectivity exists in the gas phase. One approach to quantify the gas-phase selectivity is to identify other gas-phase ligands with cation affinities similar to those of valinomycin so that equilibrium constants can be measured. This approach successfully revealed selectivity patterns in a simple crown ether-alkali metal cation system [9].

The observation of alkali cation transfer from either $18 \mathrm{C} 6$ or [2.2.2] to valinomycin and failure to observe transfer from valinomycin to a large excess of either ligand indicates that valinomycin-alkali metal complexes are more stable than the [2.2.2]-cryptate and $18 \mathrm{C} 6$ metal complexes in the gas phase. This is in direct contrast to the complexation properties observed in solution. For example, in methanol the stability constants for [2.2.2] complexes of $\mathrm{Na}^{+}, \mathrm{K}^{+}, \mathrm{Rb}^{+}$, and $\mathrm{Cs}^{+}$average $4.0 \log K$ units greater than the corresponding constants for valinomycin complexes. The same comparison in acetonitrile yields an average difference of $3.4 \log K$ units stronger for the cryptates [18].

We can speculate on the reasons for these striking differences. Factors such as differences in the number and type of donor groups in valinomycin versus the synthetic ligands probably cannot account easily for the differences, because these factors are also present in solution. Solvation can have dramatic effects on structure, which might explain the differences in solution and gas-phase behavior. Based on studies in various solvents, the conformation of valinomycin is highly solvent-dependent $[34,35]$. In nonpolar solvents (e.g., $\mathrm{CCl}_{4}$ ), all six of the amide hydrogens of valinomycin form 1-4 type intramolecular hydrogen bonds to the amide carbonyl oxygens $[34,35]$. In polar solvents (e.g., methanol), valinomycin adopts an open conformation with no intramolecular hydrogen bonds. Instead, hydrogen bonds to solvent molecules are formed $[34,35]$. The solvent molecules may be considered as competing with metal ions for the binding site(s). As a result, complex formation is destabilized in polar solvents. This is illustrated for $\mathrm{K}^{+}$complexes by stability con- 
stants measured in dioxane-water mixtures. As the dioxane fraction of the solvent increases, the stability constants increase dramatically [36].

In the gas phase the destabilizing influence of the solvent is not present. Preorganization of valinomycin due to intramolecular hydrogen bonding would therefore be expected. Both $18 \mathrm{C} 6$ and [2.2.2] lack hydrogen bond donors that could lead to this type of preorganization, so the absence of solvent should not affect the stability of their complexes to as large an extent. It is also likely that valinomycin has a higher solvation energy than either of the synthetic ionophores. To the extent that complexation requires desolvation of the ligand, valinomycin would experience a greater disadvantage in solution than the smaller less-solvated synthetic ligands. This disadvantage is also absent in gasphase complexation, leading to a relative increase in the cation affinity of valinomycin in the gas phase.

Low cation lability in the valinomycin complexes may also account for the failure of cations to transfer to the synthetic ligands, in which case the apparent stability differences might be kinetic artifacts. In the solid state, the conformations of the complexes are stabilized by six intramolecular hydrogen bonds $[37,38]$. The conformation of the gas-phase complex may also be reinforced by intramolecular hydrogen bonds that cause the structure to become very rigid, hence "locking" the central cation tightly. Alternatively, the metal ions may be "buried" deep inside the large ligand, making them inaccessible for transfer to a second ligand. This is quite similar to the behavior of crown ether-alkali metal cation complexes in which small metal ions, buried in the cavity of a crown ether molecule, do not readily bind a second ligand $[7,9]$. Self-exchange experiments with isotopically labcled ligands should shed light on this question. Preliminary experiments with crown ethers indicate that even metals such as $\mathrm{Li}^{+}$, which are quite tightly bound, are also very labile, suggesting that the high cation affinity is not a kinetic artifact. We are now beginning such studies with larger ligands like valinomycin.

Finally, if the metal-ligand interactions are primarily electrostatic in nature and therefore dominated by the polarizability of the ligand, it is not surprising that valinomycin should have relatively high alkali metal cation affinities. Crown ether-alkali metal cation complexes in the gas phase appear to be highly sensitive to these kinds of effects [9]. The polarizabilities of valinomycin, 18C6, and [2.2.2] are $112.8,25.9$, and $39.0 \AA^{3}$, respectively, calculated via the method of atomic hybrid components $[39,40]$. The polarizabilities of these three ligands follow the same order as the observed gas-phase alkali cation affinities. The ion-induced dipole interaction should be stronger in valinomycincation complexes than in complexes involving $18 \mathrm{C} 6$ or [2.2.2], and may also lead to the greater stability of valinomycin-metal ion complexes.

\section{Conclusion}

Cation attachment to valinomycin is a rapid gas-phase process, with small, charge-dense cations such as $\mathrm{Na}^{+}$ reacting more efficiently than large, diffuse cations such as $\mathrm{Cs}^{+}$. The relationship between high cation charge density and complexation efficiency appears to be general; it is evident in every system we have examined. Valinomycin has much higher intrinsic affinity for the alkali metal cations than smaller synthetic analogs such as $18 \mathrm{C} 6$ or [2.2.2]. In fact, valinomycin has the highest alkali cation affinities we have yet observed. It will be interesting and challenging to identify ligands with alkali cation affinities similar to those of valinomycin, in the hope that such ligands can be used to probe the selectivity of valinomycin toward these cations in the gas phase. As a reviewer suggests, experiments with other large cyclic peptides such as gramicidin $S$ might provide very useful comparisons that could shed light on why the alkali cation affinity of valinomycin is so large and whether cation affinities increase with ligand size and polarizability in general. Finally, solvation disfavors complexation by valinomycin relative to that by the synthetic ligands, again underscoring how trends observed in solution do not necessarily extrapolate to the simpler gas-phase environment.

\section{Acknowledgments}

We are grateful for support from the Robert A. Welch Foundation, from the donors of the Petroleum Research Fund, administered by the American Chemical Society, from the National Science Foundation (grant CHE-9221224), and from the National Science Foundation Young Investigator Program (grant CHE9357118).

\section{References}

1. Karas, M.; Bahr, U.; Giessmann, U. Mass Spectrom. Rev. 1991, $10,335-357$.

2. Smith, R. D.; Loo, J. A.; Loo, R. R. O.; Busman, M.; Udseth, H. R. Mass Spectrom. Rev. 1991, 10, 359-451.

3. Grese, R. P.; Gross, M. L. J. Am. Chem. Suc. 1990, 112, $5098-5104$

4. Teesch, L. M.; Orlando, R. C.; Adams, J. J. Am. Chem. Soc. $1991,11.3,3668-3675$

5. Teesch, L. M.; Adams, J. J. Am. Chem. Soc. 1991, 113, 812-820.

6. Alcamí, M.; Mó, O.; Yáñez, M. J. Am. Chem. Soc. 1993, 1993, 11074-11083.

7. Zhang, H.; Chu, I.-H.; Leming, S.; Dearden, D. V. J. Am. Chem. Soc. 1991, 113, 7415-7417.

8. Zhang, H.; Dearden, D. V. J. Amt. Chem. Soc. 1992, 114, $2754-2755$

9. Chu, 1.-H.; Zhang, H.; Dearden, D. V. I. Am. Chem. Soc. 1993, $115,5736-5744$.

10. Dearden, D. V.; Zhang, H.; Chu, I.-H.; Wong, P.; Chen, Q. Pure Appl. Chem. 1993, 65, 423-428.

11. Maleknia, S.; Brodbelt, J. J. Am. Chem. Soc. 1992, 114, $4295-4298$.

12. Maleknia, S.; Brodbelt, J. Rapid Commun. Mass Spectrom. 1992, $6,376-381$ 
13. Liou, C. C.; Brodbelt, J. S. J. Am. Soc. Mass Spectrom. 1992, 3, $543-548$.

14. Katritzky, A. R.; Malhotra, N.; Ramanathan, R.; Kemerait, R. C.; Zimmerman, J. A.; Eyler, J. R. Rapid Commun. Mass Spectrom. 1992, 6, 25-27.

15. Takahashi, T.; Uchiyama, A.; Yamada, K.; Lynn, B. C.; Gokel, G. W. Tetrahedron Lett. 1992, 33, 3825-3828.

16. Brezina, M.; Hofmanova-Matejkova, A.; Koryta, J. Biophys. Chem. 1974, 2, 264-268.

17. Hofmanova, A.; Koryta, J.; Brezina, M.; Ryan, T. H.; Angelis, K. Inorg. Chim. Acta 1979, 37, 135-140.

18. Izatt, R. M.; Pawlak, K.; Bradshaw, J. S.; Bruening, R. L. Chem. Rev. 1991, 91, 1721-2085.

19. Mueller, P.; Rudin, D. O. Biochem. Biophys. Res. Commun. 1967, 26, 398.

20. Gresh, N.; Etchebest, C.; De la Luz Rojas, O.; Pullman, A. Int. 1. Quantum Chem., Quantum Biol. 1981, 8, 109-116.

21. Lifson, S.; Felder, C. E.; Shanzer, A. I. Biochem. Struct. Dynam. 1984, 2, 641-661.

22. Davis, S. C.; Derrick, P. J.; Otinger, C. Z. Naturforsch. 1990, 45a, 1151-1157.

23. Sheil, M. M.; Guilhaus, M.; Derrick, P. J. Org. Mass Spectrom. 1990, 25, 671-680.

24. Curtis, J. M.; Bradley, C. D.; Derrick, P. J.; Sheil, M. M. Org. Mass Spectrom. 1992, 27, 502-507.

25. Beu, S. C.; Laude, D. A. Anal. Chem. 1992, 64, 177-180.

26. Beu, S. C.; Laude, D. A. Int. J. Mass Spectrom. Ion Processes. $1992,112,215-230$.
27. Brand, J. L.; George, S. M. Surf. Sci. 1986, 167, 341-362.

28. Hanson, C. D.; Castro, M. E.; Russell, D. H. Anal Chem. 1989, $61,2130 \cdot 2136$.

29. Rempel, D. L.; Huang, S. K.; Gross, M. L. Int. J. Mass Spectrom. Ion Processes. 1986, 70, 163.

30. Huang, S. K.; Rempel, D. I.; Gross, M. I. Int. I. Mass Spectrom. Ion Processes. 1986, 72, 15.

31. Allemann, M.; Kofel, P.; Kellerhals, H.; Wanczek, K. P. Int. J. Mass Spectrom. Ion Processes. 1987, 75, 47.

32. Marshall, A. G.; Verdun, F. R. Fourier Transforms in NMR, Optical, and Mass Spectrometry: A User's Handbook; Elsevier: Amsterdam, 1990; pp 225-278.

33. Su, T.; Bowers, M. T. In Gas Phase Ion Chemistry, Vol, 1; Bowers, M. T., Ed.; Academic: New York, 1979; pp 83-118.

34. Patel, D. J.; Tonelli, A. E. Biochemistry 1973, 12, 486-496.

35. Dobler, M. Ionophores and Their Structures; Wiley: New York, $1981 ;$ p 379.

36. Callaghan-Rose, M.; Henkens, R. W. Biochim. Biophys. Acta 1974, 372, 426-435.

37. Neupert-Laves, K.; Dobler, M. Helv. Chim. Acta 1975, 58, 432-442.

38. Duax, W. L.; Smith, G. D. Biomol. Stereodynam., Proc. Symp. 1981, 2, 311-322.

39. Miller, K. J.; Savchik, J. A. J. Am. Chem. Soc. 1979, 101, 7206-7213.

40. Miller, K. J. J. Am. Chem. Soc. 1990, 112, 8533-8542. 